\title{
Role of CT For Evaluation of Hematuria
}

\author{
Abd-Elrahman M. Kamela Mohammad T. Mahmoud ${ }^{\mathrm{a}}$, Khaled F. Zaky ${ }^{\mathrm{a}}$, \\ Mohammad A. Alsayed ${ }^{a}$, Ahmed M. Alsharkawey ${ }^{a}$
}

aDepartment of Diagnostic Radiology, Faculty of Medicine, Sohag University

\section{Abstract}

Introduction: Hematuria has a prevalence rate of $2 \%$ to $31 \%$ in the population and is therefore a common reason for imaging of the urinary tract. Hematuria has many causes ranging from urinary tract infections to carcinoma. Imaging plays a major role in the investigation of hematuria frequently determining the underlying cause and influencing management. Computed tomography (CT) is increasingly valuable tool for assessing the urinary tract in adults and children. However, its imaging capabilities, while overlapping in some respects, should be considered as complementary, as each technique offers specific advantages and disadvantages both in actual inherent qualities of the technique and in specific patients and with a specific diagnostic question. The use of CT should therefore be tailored to the patient and the clinical question.

Aim of the work: Evaluation of CT as imaging techniques for assessment and diagnosis of causes of hematuria and to study the CT features of various etiologies causing it.

Patients and Methods: A prospective observational study included 42 cases have hematuria (gross \& microscopic) were examined using MDCT. They are presented to the outpatient clinic of the urology and radiology department at Sohag university hospital between September 2015 and September 2018. A written consent was taken from the patients included in the study. An approval was taken from the scientific ethical committee before starting in the protocol of this study.

Results: All causes of hematuria of urologic origin were almost covered using multidetector CT that was very conclusive compared to the previous traditional examinations that the patient had. These images have met good acceptance by the referring urologist that was comparable to endoscopic and surgical results, proving that MDCT can give accurate evaluation for hematuria instead of performing multiple traditional studies that may expose the patient to frequent contrast material \& to frequent radiation exposure. We recommend CT as an initial investigation of hematuria and appropriate protocol should be tailored for each patient.

Conclusion: CT can diagnose wide spectrum of diseases associated with hematuria with good accuracy and considered the initial and comprehensive imaging modality for evaluation of hematuria.

Key words: Hematuria, CT.

\section{Introduction}

Hematuria has a prevalence rate of $2 \%$ to $31 \%$ in the population and is therefore a common reason for imaging of the urinary tract. Hematuria has many causes ranging from urinary tract infections to carcinoma. Imaging plays a major role in the investigation of hematuria frequently determining the underlying cause and influencing management(Sharp et al., 2013).
Hematuria is characterized as either microscopic or gross hematuria. Microscopic is defined by the American Urological Association as 3 or more red blood cells per high power field on microscopic evaluation of urinary sediment from "one properly collected, non-contaminated urinalysis. Gross hematuria is defined as hematuria visible to the physician or patient(Davis et al., 2012).Gross hematuria has a high association with malignancy of up to $30-40 \%$, and 
therefore all patients with gross hematuria should have a full urologic workup. Conversely, patients with microscopic have a low risk of malignancy ranging from 2.6-4\% (Sharp et al., 2013).

Currently there is no universal agreement about the optimal imaging work up of hematuria. The choice of modality to image the urinary tract will depend on individual patient factors such as age, the presence of risk factors for malignancy, renal function, a history of calculus disease and pregnancy, and other factors, such as local policy and practice, cost

\section{Aim of the work:}

This study aims at evaluation of CT as imaging techniques for assessment and diagnosis of causes of hematuria and to

\section{Patients and Methods:}

A prospective observational study included 42 cases have hematuria (gross \& microscopic) were examined using MDCT. They are presented to the outpatient clinic of the urology and radiology department at Sohag university hospital between September 2015 and September 2018. A written consent was taken from the patients included in the study. An approval was taken from the scientific ethical committee before starting in the protocol of this study.

Using 8-detectors GE Light Speed CT scanner (Milwaukee-USA).

Making use of already available patient data including history, clinical examination, previous radiological MDCT protocols:

-In cases where UT stones are suspected, just thin slices non-enhanced cuts are obtained.

-In cases suspected other causes for hematuria apart from UT stones, three effectiveness and availability of resources(Moloney et al., 2014).

Computed tomography (CT) is increasingly valuable tool for assessing the urinary tract in adults and children. However, its imaging capabilities, while overlapping in some respects, should be considered as complementary, as each technique offers specific advantages and disadvantages both in actual inherent qualities of the technique and in specific patients and with a specific diagnostic question. The use of CT should therefore be tailored to the patient and the clinical question (Melanie 2011).

study the CT features of various etiologies causing it.

examination, to select the suitable and appropriate protocol.

Patient evaluation included: personal data, clinical findings, other imaging modalities, laboratory investigations such as urine analysis and renal functions tests.

\section{Inclusion criteria.}

Patients presenting with macroscopic hematuria

Patients with documented microscopic hematuria

Patients with hematuria who were suspected to have urinary tract pathology on other modalities.

\section{Exclusion criteria.}

Patients with non-urologic causes of hematuria.

phases CTU was done unless modified according to the case, for example suspecting renal mass lesion corticomedullary phase may be added and for special case suspecting post-operative ureteric injury only excretory phase was 
-Phases are detailed as the following Table (1): 3 phases CTU protocol:(Michel et al., 2004)

\begin{tabular}{|c|c|c|c|c|c|c|}
\hline & $\begin{array}{l}\text { Detector } \\
\text { collimation }\end{array}$ & $\begin{array}{l}\text { Section } \\
\text { thickness }\end{array}$ & $\begin{array}{l}\text { Reconstruct } \\
\text { ion interval }\end{array}$ & Pitch & $\mathrm{Kv} / \mathrm{Ma}$ & Timing \\
\hline $\begin{array}{l}\text { Non- } \\
\text { contrast } \\
\text { phase }\end{array}$ & $4 \times 5$ & $2.5-5 \mathrm{~mm}$ & 4 & $1.5: 1$ & $120 / 30$ & \\
\hline $\begin{array}{l}\text { Nephrograp } \\
\text { hic phase }\end{array}$ & $4 \times 2.5$ & $2.5 \mathrm{~mm}$ & 2 & $1.5: 1$ & $120 / 200$ & $\begin{array}{l}90 \text { to } 120 \text { s after } \\
\text { contrast injection }\end{array}$ \\
\hline $\begin{array}{l}\text { Excretory } \\
\text { phase }\end{array}$ & $4 \times 2.5$ & $2.5 \mathrm{~mm}$ & 2 & $1.5: 1$ & $120 / 200$ & $\begin{array}{l}5 \text { to } 10 \mathrm{~m} \text { after } \\
\text { contrast injection }\end{array}$ \\
\hline
\end{tabular}

\section{Results}

42 patients of different age groups underwent CT, for evaluation of hematuria, most of them come with gross hematuria, and others come with other urological manifestation and during initial evaluation by urine analysis revealed microscopic hematuria and radiological evaluation is requested.

Nearly all causes of hematuria can be adequately evaluated by $\mathrm{CT}$ imaging either urolithiasis, neoplastic, inflammatory or congenital anomalies, also for trauma it has a major role where it fast and accurate examination suitable for those cases. Also CT examinations can be tailored according to the suspected etiology and patients conditions to give the best results and decreased the hazards of exposure to radiation. 
Table (2): CT findings in our study.

\begin{tabular}{|c|c|c|}
\hline \multicolumn{1}{|c|}{ CT findings } & No. & \% \\
\hline \hline Stones & $\mathbf{1 5}$ & $\mathbf{3 4 . 8}$ \\
\hline Renal stones & 6 & 14 \\
\hline Ureteric stone with hydro nephrosis & 9 & 20.9 \\
\hline Trauma & $\mathbf{7}$ & $\mathbf{1 6 . 2}$ \\
\hline Renal laceration and hematoma & 4 & 9.3 \\
\hline Ureteric injury & 1 & 2.3 \\
\hline Gun shots & 2 & 4.6 \\
\hline Mass $\quad 15$ & $\mathbf{3 4 . 8}$ \\
\hline Renal mass & 6 & 14 \\
\hline UB malignant masses & 8 & 8.7 \\
\hline Ureteric mass & 1 & 2.3 \\
\hline Congenital anomalies & $\mathbf{2}$ & $\mathbf{4 . 6}$ \\
\hline Horseshoe kidney (no other findings explain hematuria) & 1 & 2.3 \\
\hline PCKD & 1 & 2.3 \\
\hline Inflammatory & 1 & $\mathbf{6 . 9}$ \\
\hline Bilateral Perinephric abscess & 1 & 2.3 \\
\hline Renal TB: Bilateral small sized kidney with coarse & & 2.3 \\
\hline RT Emphysematous pyelonephritis & 1 & \\
\hline
\end{tabular}

\section{Illustrative cases:}

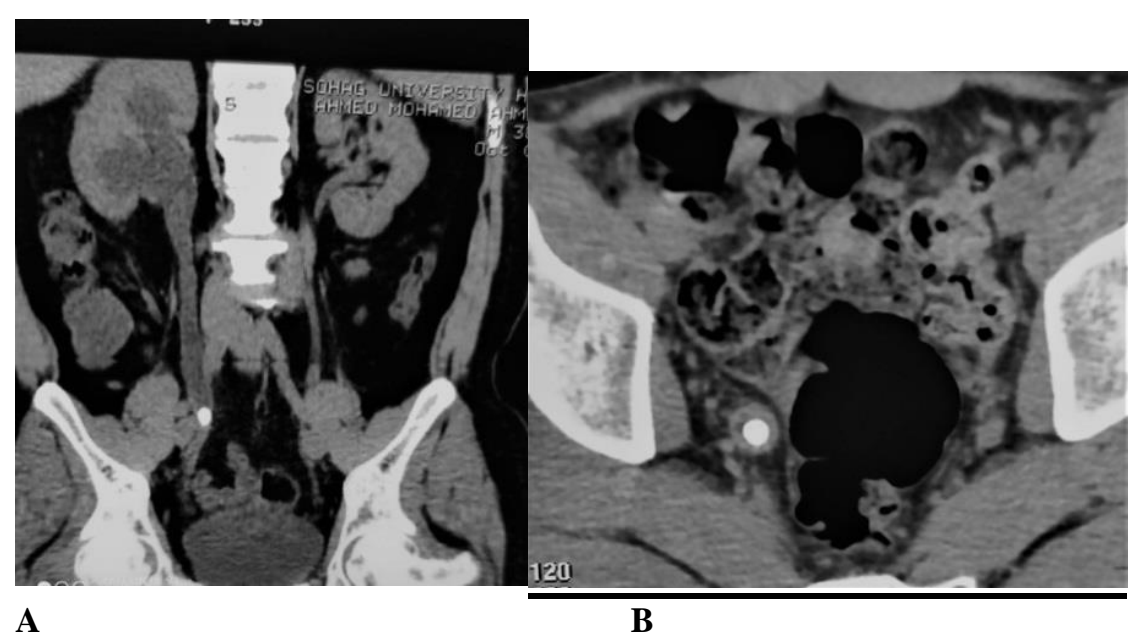

Figure (1) RT ureteric calculus:(A) coronal and (B) axial images. 
Male patient 28 ys old come with gross hematuria and RT colicky pain, unenhanced CTU coronal and axial cuts revealed RT hydronephrosis due to stone at middle third of the RT ureter about $(2 \times 1) \mathrm{cm}$ and $\mathrm{HU}$ about 1100.

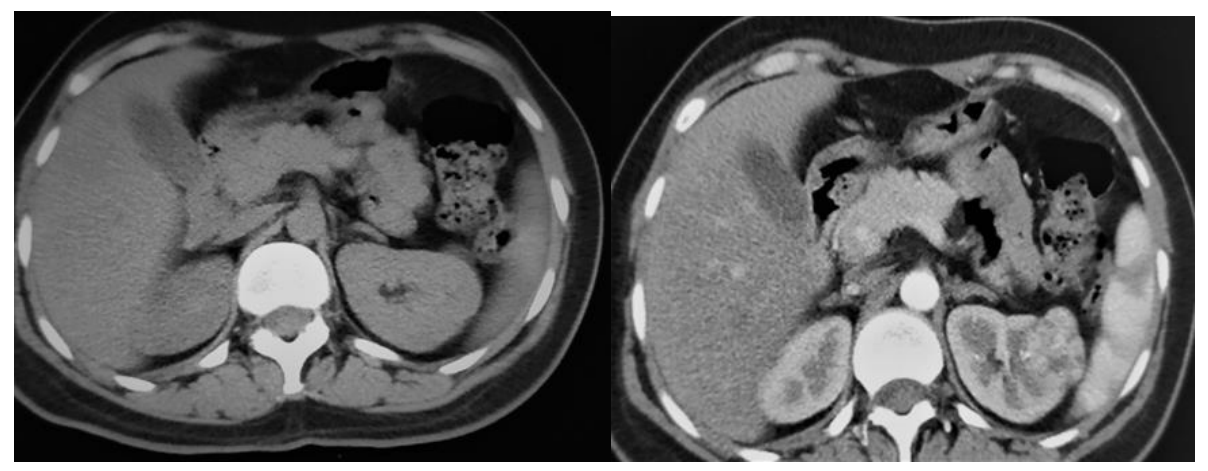

A

B

Figure (2) LT renal mass:(A) axial precontrast and (B) axial arterial phase images:

Female patient 45 ys old come with gross hematuria, enhanced CTU was done, axial precontrast image revealed focal bulge at LT side of the LT kidney and axial corticomedullary phase image revealed intensely enhanced mass lesion at the site of the focal bulge, about $(3.5 \times 3) \mathrm{cm}$, partial nephrectomy and histopathology revealed RCC.

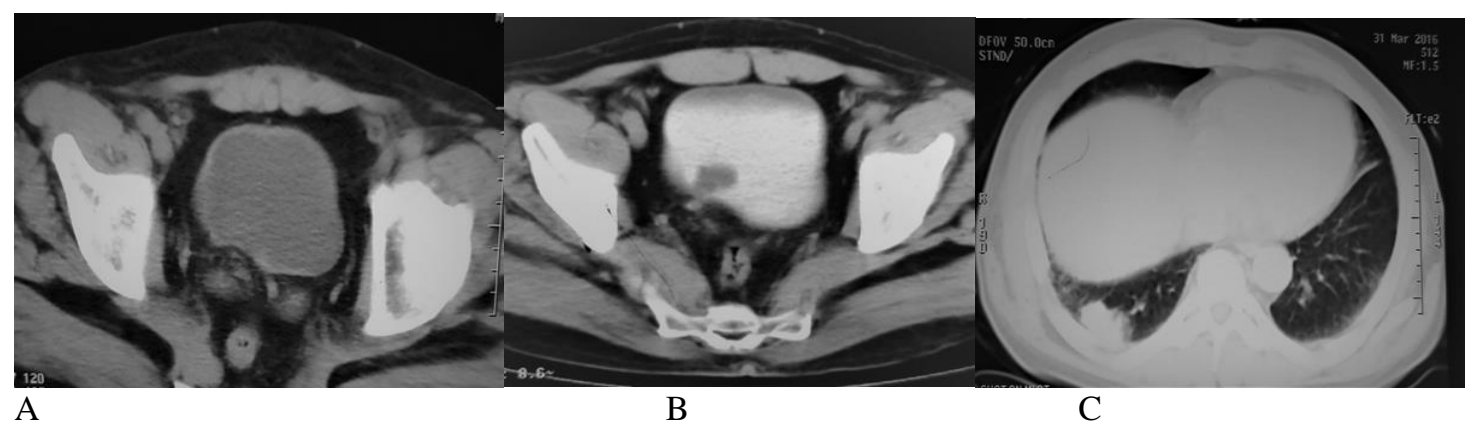

Figure 3 Urinary bladder mass: (A) axial precontrast, (B) axial delayed phase images and $(\mathrm{C})$ axial chest image:

Male patient 60 ys presented with gross hematuria, enhanced CTU was done revealed axial precontrast image show focal UB wall retraction at RT side of the base, no visible mass lesion, axial delayed phase image revealed polypoidal small mass lesion at the site of the retraction about $(3 \times 2.5) \mathrm{cm}$, axial image of the lower part of the chest show RT basal nodule in favor of metastatic deposite where complete CT chest was done show further 3 small nodules.

\section{Discussion}

This is a prospective observational study that was performed over a time interval aiming to show value of MDCT in diagnosis of urological disorder associated with hematuria.
According to the patient conditions \& suspected lesion to be found (depending on previous radiological examination \& patient clinical data), 
choice and protocol of the study was tailored.

The principal indication for CT urography is the investigation of hematuria. Rapid developments in multidetector CT technology make multiplanar review of isotropic datasets possible providing high-resolution CT urography studies. The high diagnostic accuracy of unenhanced CT for stones, nephrographic phase CT for renal masses and excretory phase CT for UUT-UCC (compared to other techniques) makes CT urography the preferred initial imaging investigation for patients presenting with hematuria (Cowan et al., 2007).

As regard urolithiasis, unenhanced helical CT is highly sensitive (up to $98 \%)$ and specific (96-100\%) in diagnosing urolithiasis, and is the imaging modality of choice for the initial evaluation of patients with suspected urinary stones (Dhar and Denstedt 2009). Our study showed that in patients with renal colic and hematuria (suspected to have urinary stone), unenhanced MDCT is a very sensitive method about $100 \%$ for detecting any UT stones that may not be detected by other imaging modalities.

As regard congenital anomalies: Most congenital anomalies of urinary tract can be appreciated with MDCTU. CT arteriography (CTA) is sometimes performed in instances of known horseshoe kidney when surgery is being planned. This is to optimally delineate the multiple vessels that often supply these kidneys before surgery is undertaken. In our study CT accurately diagnose a case of horseshoe kidney and surgery was not planned for.

As regard renal masses: The detection rate of renal masses has increased in the last decades owing to the widespread use of CT. Therefore, an accurate characterization of renal masses is essential to ensure appropriate case management. Renal masses can be divided into cystic and solid lesions. CT enhancing masses are classified as solid or complex cystic. Eighty-five percent of expansive solid masses are malignant. Therefore, a solid, enhancing mass must be considered malignant unless proved otherwise. Renal cell carcinoma (RCC) is the most common malignant tumor (Srougi et al., 2009) as in our study where the 4 malignant featuring renal masses were RCC. Leo PallweinPrettner et al., 2009 compared CT and MRI in the evaluation of cystic renal masses by using the Bosniak classification system. CT and MRI findings were similar for most cystic renal masses. in our study one case showing multilocular cystic renal lesion by CT and classified as Bosniak III.

At CT ninety percent of clear cell RCCs are hypervascular with a heterogeneous enhancing pattern of mixed enhancing solid soft-tissue components and low-attenuation necrotic or cystic areas. All cases of our study are hypervascular. Also Clear cell carcinomas can be predominantly cystic. Renal vein tumor thrombus can be seen with aggressive higher stage tumors (Leo Pallwein-Prettner et al., 2009). In a case of our series with RCC, thrombosed right renal vein with extension into the IVC seen.

Angiomyolipomas are hamartomas containing varying proportions of fat, smooth muscle and thick-walled blood vessels. Tumors larger than $4 \mathrm{~cm}$ carry an increased risk of potentially lifethreatening hemorrhage and heavy hematuria (Wunderlich syndrome), which has been reported in up to $10 \%$ of these patients (Simpfendorfer et al., 2009). CT Lipid-containing angiomyolipoma can be easily detected with CT. In our series a case was diagnosed at CT as angiomyolipoma, which was bilateral and multiple with also hepatic angiomyolipoma, 
confirmed to be a case of TS. However, angiomyolipoma may contain very small quantities of fat, which can be overlooked if the mass is not carefully evaluated. When a small amount of fat is suspected in a renal mass, an unenhanced CT examination with thin sections and, if necessary, a pixel analysis is the most sensitive test (Simpfendorfer et al., 2009).

As regard urothelial carcinoma, the diagnostic performance of CT is better than is commonly thought: In a study by Turney et al., 2006, 200 patients with hematuria underwent both cystoscopy and CT urography. Bladder malignancy was found in $24 \%$ of the patients, and the sensitivity and specificity of CT urography were $93 \%$ and $99 \%$. In our study sensitivity reach $100 \%$.

As regard accuracy of CT in local staging of UB cancer: Conventional CT was only moderately accurate in local staging of bladder cancer; cystoscopy and pathologic staging remain the standards of reference. The accuracy of CTU in the local staging of bladder cancer is only $40 \%-60 \%$ (Verma et al., 2012). In our study sensitivity of CT is $66.7 \%$.

As regard traumatic injury: in our study there is 5 cases renal trauma, 4 cases blunt tauma (MCA or falling from height), one case penetrating trauma (gun shots), CT accurately diagnose and grading the lesions where ranged

\section{Conclusion:}

All causes of hematuria of urologic origin were almost covered using multidetector CT that was very conclusive compared to the previous traditional examinations that the patient had. These images have met good

\section{References:}

1.Sharp VJ, Barnes KT, Erickson BA (2013). Assessment of asymptomatic microscopic hematuria in adults. American family physician. 88(11):747-54. from grade I to grade IV with very comparable post-operative results in cases needed surgery, so perfect management and save kidney as much as possible was achieved. One of our cases showing iatrogenic uretric injury after abdominal exploration and excretory phase CTU was done revealed uretric injury and contrast leakage at that part.

As regard inflammatory lesions: Most commonly, infection of the urinary tract presents clinically and is treated empirically. However, in a few more complicated cases, imaging will be performed to look for complications, such as abscess formation or pyelonephritis, within the kidneys and for the presence of underlying contributory factors, such as stone disease or previously undiagnosed structural anomalies. CT may add further information regarding, for example, abscess rupture or retroperitoneal fluid (or pus) collections. CT is useful in showing calcification and extrarenal complications in xanthogranulomatous pyelonephritis (Melanie 2011). In our study, one case showing right emphysematous pyelonephritis complicated with lumber abscess and another case with bilateral renal atrophy and patchy calcifications suggesting renal TB and clinical \& laboratory findings highly suggested that diagnosis.

acceptance by the referring urologist that was comparable to endoscopic and surgical results, We recommend CT as acomprehensive investigation for hematuria.

2.Davis R, Jones JS, Barocas DA, Castle EP, Lang EK, Leveillee RJ, et al., (2012). Diagnosis, evaluation and follow-up of asymptomatic microhematuria $(\mathrm{AMH})$ in adults: AUA 
guideline. The Journal of urology. 188(6 Suppl):2473-81.

3.Moloney F, Murphy KP, Twomey M, O'Connor OJ, Maher MM, (2014).Haematuria: an imaging guide. Advances in urology.

4.Melanie P Hiorns, (2011). Imaging of the urinary tract: the role of CT and MRI. Pediatric nephrology. 26(1):5968.

5.Cowan NC, Turney BW, Taylor NJ, McCarthy CL, Crew JP, (2007).Multidetector computed tomography urography for diagnosing upper urinary tract urothelialtumour. BJU international. 99(6):1363-70.

6.Dhar M, Denstedt JD, (2009). Imaging in diagnosis, treatment, and follow-up of stone patients. Advances in chronic kidney disease. 16(1):39-47.

7.Srougi V, Kato RB, Salvatore FA, Ayres PP, Dall'Oglio MF,(2009). Incidence of benign lesions according to tumor size in solid renal masses. International braz j urol. 35(4):427-31. 8.Leo Pallwein-Prettner, Flöry D, Rotter CR, Pogner K, Syré G, Fellner C, et al., (2011). Assessment and characterisation of common renal masses with $\mathrm{CT}$ and MRI. Insights into imaging. (5):543-56.

9.Simpfendorfer C, Herts BR, MottaRamirez GA, Lockwood DS, Zhou M, Leiber M, et al., (2009).Angiomyolipoma with minimal fat on MDCT: can counts of negativeattenuation pixels aid diagnosis? American Journal of Roentgenology. 192(2):438-43.

10.Turney BW, Willatt JM, Nixon D, Crew JP, Cowan NC, (2006). Computed tomography urography for diagnosing bladder cancer. BJU international. 98(2):345-8.

11.Verma S, Rajesh A, Prasad SR, Gaitonde K, Lall CG, Mouraviev V, et al., (2012). Urinary bladder cancer: role of MR imaging. Radiographics : a review publication of the Radiological Society of North America, Inc. 32(2):371-87.

Michael M. Maher, Mannudeep K. Kalra, Stefania Rizzo, Peter R. Mueller, Sanjay Saini, (2004). Multidetector CT Urography in Imaging of the Urinary Tract in Patients with Hematuria. Korean J Radiol5:1-10 